PROCEEDINGS OF THE

AMERICAN MATHEMATICAL SOCIETY

Volume 131, Number 1, Pages 275-284

S 0002-9939(02)06658-3

Article electronically published on June 3, 2002

\title{
INTERPOLATION OPERATORS ASSOCIATED WITH SUB-FRAME SETS
}

\author{
DEGUANG HAN
}

(Communicated by David R. Larson)

\begin{abstract}
Interpolation operators associated with wavelets sets introduced by Dai and Larson play an important role in their operator algebraic approach to wavelet theory. These operators are also related to the von Neumann subalgebras in the "local commutant" space, which provides the parametrizations of wavelets. It is a particularly interesting question of how to construct operators which are in the local commutant but not in the commutant. Motivated by some questions about interpolation family and $\mathrm{C}^{*}$-algebras in the local commutant, we investigate the interpolation partial isometry operators induced by sub-frame sets. In particular we introduce the $2 \pi$-congruence domain of the associated mapping between two sub-frame sets and use it to characterize these partial isometries in the local commutant. As an application, we obtain that if two wavelet sets have the same $2 \pi$-congruence domain, then one is a multiresolution analysis (MRA) wavelet set which implies that the other is also an MRA wavelet set.
\end{abstract}

\section{INTRODUCTION}

An orthonormal wavelet (or wavelet for short) is a function $\psi \in L^{2}(\mathbb{R})$ such that $\left\{\psi_{j, k}: j, k \in \mathbb{Z}\right\}$ forms an orthonormal basis, where $\psi_{j, k}(x)=2^{\frac{j}{2}} \psi\left(2^{j} x-k\right)$. If $T$ and $D$ are the translation and dilation unitary operators, respectively, on $L^{2}(\mathbb{R})$ defined by $(T f)(t)=f(t-1)$ and $(D f)(t)=\sqrt{2} f(2 t)$, then $\psi$ is a wavelet if and only if $\left\{D^{j} T^{k} \psi: j, k \in \mathbb{Z}\right\}$ is an orthonormal basis for $L^{2}(\mathbb{R})$.

Let $\mathcal{F}$ be the Fourier-Plancherel transform on $L^{2}(\mathbb{R})$. Then $\mathcal{F}$ is a unitary transformation and if $f, g \in L^{1}(\mathbb{R}) \cap L^{2}(\mathbb{R})$, we have

$$
(\mathcal{F} f)(s)=\frac{1}{\sqrt{2 \pi}} \int_{\mathbb{R}} e^{-i s t} f(t) d t=: \hat{f}(s)
$$

and

$$
\left(\mathcal{F}^{-1} g\right)(t)=\frac{1}{\sqrt{2 \pi}} \int_{\mathbb{R}} e^{i s t} g(s) d s .
$$

Let $\hat{D}=\mathcal{F} D \mathcal{F}^{-1}$ and $\hat{T}=\mathcal{F} T \mathcal{F}^{-1}$. Then $\hat{D}=D^{-1}=D^{*}$ and $\hat{T}=M_{e^{-i s}}$, where for $h \in L^{\infty}(\mathbb{R}), M_{h}$ denotes the multiplication operator $f \rightarrow h f, \quad f \in L^{2}(\mathbb{R})$.

Received by the editors February 1, 2001 and, in revised form, September 10, 2001.

2000 Mathematics Subject Classification. Primary 42C15, 47B38.

Key words and phrases. Wavelet, sub-frame set, interpolation operators, congruence domain, multiresolution analysis, MRA wavelet set. 
Following Dai and Larson $([\overline{\mathrm{DL}}])$, a measurable set $E$ of $\mathbb{R}$ is called a wavelet set if the inverse Fourier transform of $\frac{1}{\sqrt{2 \pi}} \chi_{E}$ is a wavelet. Such a wavelet is called an s-elementary wavelet in [DL] and minimally-supported frequencies wavelet (or MSF wavelet for short) in [HW]. Shannon's wavelet $\psi_{S}$ is a simple example of MSF wavelet since $\hat{\psi_{S}}=\frac{1}{\sqrt{2 \pi}} \chi_{E}$ with $E=(-2 \pi,-\pi] \cup(\pi, 2 \pi]$. There are many others. Indeed it was proved in $\mathrm{Sp}$ that all the s-elementary wavelets are path-connected in norm $(\underline{\mathrm{Sp}} \mid)$, and the collection of all the wavelet sets forms the Borel algebra $($ DLS $]$.

Let $\mathcal{S}$ be a subset of the algebra of all bounded linear operators on a Hilbert space $H$ and $x \in H$. Recall from DL that the local commutant of $\mathcal{S}$ at $x$ is defined by

$$
C_{x}(\mathcal{S})=\{T \in B(H):(T S-S T) x=0, s \in \mathcal{S}\} .
$$

When $\mathcal{S}=\left\{D^{n} T^{m}: m, n \in \mathbb{Z}\right\}$ (resp. $\mathcal{S}=\left\{\hat{D}^{n} \hat{T}^{m}: m, n \in \mathbb{Z}\right\}$ ), we write $C_{\psi}(\mathcal{S})=C_{\psi}(D, T)$ (resp. $\left.C_{\psi}(\hat{D}, \hat{T})\right)$. The connection between the wavelets and the local commutant at a fixed wavelt $\psi$ is that there is a one-to-one correspondence in the sense that for each wavelet $\varphi$, there is a unique unitary $U \in C_{\psi}(D, T)$ such that $\varphi=U \psi$, and vice versa. It is known ([DL], [La $)$ that there exist many nontrivial von Neumann algebras in $C_{\psi}(D, T)$ that induce the interesting interpolation theory for wavelets. In particular, Meyer's wavelet family can be obtained by interpolating two simple wavelet sets. Although many structure properties for local commutants have been extensively investigated in [DL, it is still far from clear to understand the structure of $C_{\psi}(D, T)$. In particular it is not even known whether a local commutant contains von Neumann algebras of infinite type. In this paper we investigate a special class of partial isometry operators in $C_{\psi}(D, T)$ that are induced by some measurable sets. In the case that these sets are wavelet sets, we get the interpolation unitary operators which are important in deriving new wavelets by interpolating two simple wavelet sets ([DL], La $)$. We will characterize when these operators belong to the local commutant. As an application, we get a special result connecting MRA wavelet sets (for the definition see section 2), stating that if two wavelet sets form an interpolation pair, then one is an MRA wavelet set if and only if the other is. We hope that our investigation will eventually lead to some progress in constructing some interesting operator algebras in a given local commutant.

Some of the ideas in this paper were developed when the author was a graduate student at Texas A\&M University, and he would like to thank Professor D. Larson for many stimulating discussions about the interpolation families of wavelets.

\section{INTERPOLATION MAPPINGS AND OPERATORS}

Let $E$ and $F$ be two measurable subsets of $\mathbb{R}$. We say that $E$ is $2 \pi$-congruent to $F$ (denoted by $E \sim_{2 \pi} F$ ) if there exists a measurable partition $\left\{E_{n}\right\}$ of $E$ such that $\left\{E_{n}+2 n \pi\right\}$ is a partition of $F$. By a partition of $E$ we mean $\bigcup_{n \in \mathbb{Z}} E_{n}=E$ and $\left\{E_{n}\right\}$ is a disjoint family in the sense that $E_{n} \cap E_{m}$ has measure zero when $m \neq n$.

Recall that a sequence $\left\{f_{j}\right\}$ in a Hilbert space $H$ is called a normalized tight frame for $H$ if the following holds:

$$
\|f\|^{2}=\sum_{j}\left|\left\langle f, f_{j}\right\rangle\right|^{2}, \quad f \in H .
$$


A measurable set $E$ is called a sub-frame set if $E$ is $2 \pi$-translation congruent to a subset of $\left[0,2 \pi\right.$ ) and $\left\{2^{n} E: n \in \mathbb{Z}\right\}$ is a disjoint family. It is known (cf. DDG], [DDGH]) that $E$ is a sub-frame set if and only if $\left\{D^{m} T^{n} \psi_{E}: m, n \in \mathbb{Z}\right\}$ is a normalized tight frame for the closed subspace generated by this sequence, where $\hat{\psi}_{E}=\frac{1}{\sqrt{2 \pi}} \chi_{E}$. The term frame set is used in [HL] when this subspace is the whole space $L^{2}(\mathbb{R})$. Therefore a sub-frame set $E$ is a frame set if and only if $\bigcup_{n \in \mathbb{Z}} 2^{n} E=\mathbb{R}$, while it is a wavelet set if and only if both $\bigcup_{n \in \mathbb{Z}} 2^{n} E=\mathbb{R}$ and $\mu(E)=2 \pi$, where $\mu$ is the Lebesgue measure on $\mathbb{R}$ (cf. [HL]).

For any subset $G$ of $\mathbb{R}$ we write $\tilde{G}=\bigcup_{n \in \mathbb{Z}} 2^{n} G$. Let $E$ and $F$ be two sub-frame sets such that $E \sim_{2 \pi} F$. Then $\sigma_{E}^{F}: E \rightarrow F$, uniquely defined by $2 \pi$-translation congruence, can be extended to $\tilde{E}$ by

$$
\sigma_{E}^{F}(x):=2^{n} \sigma_{E}^{F}\left(2^{-n} x\right), \quad x \in 2^{n} E .
$$

It is easy to check that $\sigma_{E}^{F}$ is a measure preserving mapping from $\tilde{E}$ onto $\tilde{F}$. We will call it the interpolation mapping from $E$ to $F$. The associated interpolation operator $U_{E}^{F}$ on $L^{2}(\mathbb{R})$ is defined by

$$
\left(U_{E}^{F} f\right)(s):=\left\{\begin{aligned}
f\left(\sigma_{F}^{E}(s)\right), & s \in \tilde{F}, \\
0, & s \notin \tilde{F} .
\end{aligned}\right.
$$

The following lemma summarizes some properties which will be used later.

Lemma 2.1. Let $E$ and $F$ be two sub-frame sets. Then:

(i) $\left(U_{E}^{F}\right)^{*}=U_{F}^{E}$.

(ii) $U_{E}^{F}$ is a partial isometry with initial subspace $L^{2}(\tilde{E})$ and final subspace $L^{2}(\tilde{F})$.

(iii $U_{E}^{F}=V \oplus 0$ for some unitary operator $V$ on some subspace of $L^{2}(\mathbb{R})$ if and only if $\tilde{E}=\tilde{F}$.

(iv) $U_{E}^{F}=\left(U_{E}^{F}\right)^{*}$ if and only if $\left(\sigma_{E}^{F}\right)^{2}=i d$ on $\tilde{E}$ and $\tilde{E}=\tilde{F}$.

(v) $U_{E}^{F}$ is a projection if and only if $E=F$.

Proof. (i) For any $f, g \in L^{2}(\mathbb{R})$, we have

$$
\begin{aligned}
\left\langle U_{F}^{E} f, g\right\rangle & =\int_{\mathbb{R}}\left(U_{F}^{E} f\right)(s) \overline{g(s)} d s \\
& =\int_{\tilde{E}} f\left(\sigma_{E}^{F}(s) \overline{g(s)} d s\right. \\
& =\int_{F \sim} f(t) \overline{g\left(\sigma_{F}^{E}(t)\right)} d t \\
& =\int_{\mathbb{R}} f(t) \overline{\left(U_{E}^{F} g\right)(t)} d t=\left\langle f, U_{E}^{F} g\right\rangle .
\end{aligned}
$$

Thus $\left(U_{F}^{E}\right)^{*}=U_{E}^{F}$.

(ii) Let $f \in L^{2}(\tilde{F})$. Since $\left(\sigma_{E}^{F} \circ \sigma_{F}^{E}\right)(s)=s$ on $F$, it follows from the definitions of $U_{E}^{F}$ and $U_{F}^{E}$ that $\left(U_{E}^{F} U_{F}^{E} f\right)(s)=f(s)$ and $\left(U_{E}^{F} U_{F}^{E}\right) g=0$ when $g \in L^{2}(\mathbb{R} \backslash \tilde{F})$. Thus (ii) follows from (i).

(iii) follows from (i).

(iv) The sufficiency follows from the definition of $U_{E}^{F}$ and (i). For the necessity, by (iii), we have that $\tilde{E}=\tilde{F}$ and $U_{E}^{F}=V \oplus 0$ with $V^{*}=V$. Thus $V^{2}=I$, which 
implies that if $f \in L^{2}(\tilde{E})$, then $\left(U_{E}^{F}\right)^{2} f=f$. That is

$$
f(s)=\left\{\begin{aligned}
f\left(\left(\sigma_{F}^{E}\right)^{2}(s)\right), & s \in \tilde{F}, \\
0, & s \notin \tilde{F} .
\end{aligned}\right.
$$

Thus $\left(\sigma_{F}^{E}\right)^{2}=i d$ on $\tilde{F}$ since $\tilde{E}=\tilde{F}$. Therefore $\left(\sigma_{E}^{F}\right)^{2}=i d$ on $\tilde{E}$.

(v) Suppose that $U_{E}^{F}$ is a projection. Then, by (iv), we have $U_{E}^{F} f=f$ for all $f \in L^{2}(\tilde{E})$. Thus $\sigma_{F}^{E}(s)=s$ if $s \in F$, which implies that $F \subseteq E$. Similarly $E \subseteq F$.

We will first characterize $E$ and $F$ so that $U_{E}^{F}$ belongs to $C_{\psi}(\hat{D}, \hat{T})$, where $\hat{\psi}=\chi_{G}$ for some measurable set $G$ (in what follows we also use $C_{G}(\hat{D}, \hat{T})$ to denote $\left.C_{\psi}(\hat{D}, \hat{T})\right)$. For this purpose we introduce the following:

Definition 2.1. The domain of $2 \pi$-congruence of $\sigma_{E}^{F}$, denoted by $\mathcal{D}_{E}^{F}$, is the set of all points $s \in \tilde{E}$ for which $\sigma_{E}^{F}(s)-s$ is an integral multiple of $2 \pi$.

Theorem 2.2. Let $G$ be any measurable subset of $\mathbb{R}$ such that $\mu(G)<\infty$. Then $U_{E}^{F} \in C_{G}(\hat{D}, \hat{T})$ if and only if $G \cap \tilde{E} \subseteq \mathcal{D}_{E}^{F}$.

Proof. For any $n, m \in \mathbb{Z}$, we have

$$
\left(\hat{D}^{n} \hat{T}^{m} \chi_{G}\right)(s)=2^{-\frac{n}{2}} e^{i \frac{m}{2^{n}} s} \chi_{2^{n} G}(s) .
$$

Thus

$$
\begin{aligned}
U_{E}^{F}\left(\hat{D}^{n} \hat{T}^{m} \chi_{G}\right)(s) & =\left\{\begin{aligned}
2^{-\frac{n}{2}} e^{i \frac{m}{2^{n}} \sigma_{F}^{E}(s)} \chi_{2^{n} G}\left(\sigma_{F}^{E}(s)\right), & s \in \tilde{F}, \\
0, & s \notin \tilde{F} .
\end{aligned}\right. \\
& =\left\{\begin{aligned}
2^{-\frac{n}{2}} e^{i \frac{m}{2^{n}} \sigma_{F}^{E}(s)} \chi_{\sigma_{E}^{F}\left(2^{n} G \cap \tilde{E}\right)}(s), & s \in \tilde{F}, \\
0, & s \notin \tilde{F}
\end{aligned}\right. \\
& =2^{-\frac{n}{2}} e^{i \frac{m}{2^{n}} \sigma_{F}^{E}(s)} \chi_{\sigma_{E}^{F}\left(2^{n} G \cap \tilde{E}\right)}(s)
\end{aligned}
$$

and

$$
\begin{aligned}
\left(\hat{D}^{n} \hat{T}^{m} U_{E}^{F} \chi_{G}\right)(s) & =\hat{D}^{n} \hat{T}^{m}\left\{\begin{aligned}
\chi_{G}\left(\sigma_{F}^{E}(s)\right), & s \in \tilde{F}, \\
0, & s \notin \tilde{F}
\end{aligned}\right. \\
& =\hat{D}^{n} \hat{T}^{m}\left\{\begin{aligned}
\chi_{\sigma_{E}^{F}(G \cap \tilde{E})}(s), & s \in \tilde{F} \\
0, & s \notin \tilde{F}
\end{aligned}\right. \\
& =\hat{D}^{n} \hat{T}^{m} \chi_{\sigma_{E}^{F}(G \cap \tilde{E})}(s) \\
& =2^{-\frac{n}{2}} e^{i \frac{m}{2^{n}} s} \chi_{\sigma_{E}^{F}(G \cap \tilde{E})}\left(2^{-n} s\right) \\
& =2^{-\frac{n}{2}} e^{i \frac{m}{2^{n}} s} \chi_{2^{n} \sigma_{E}^{F}(G \cap \tilde{E})}(s) \\
& =2^{-\frac{n}{2}} e^{i \frac{m}{2^{n}} s} \chi_{\sigma_{E}^{F}\left(2^{n} G \cap \tilde{E}\right)}(s)
\end{aligned}
$$

since $2^{n} \tilde{E}=\tilde{E}$ and $2^{n} \sigma_{E}^{F}(x)=\sigma_{E}^{F}\left(2^{n} x\right)$.

Thus we have that $U_{E}^{F} \in C_{G}(\hat{D}, \hat{T})$ if and only if for any $n, m \in \mathbb{Z}$ and any $s \in 2^{n} G \cap \tilde{E}$ (hence $\sigma_{E}^{F}(s) \in \sigma_{E}^{F}\left(2^{n} G \cap \tilde{E}\right)$ ), there is an integer $k$ such that $\frac{m}{2^{n}} s=$ $\frac{m}{2^{n}} \sigma_{E}^{F}(s)+2 k \pi$.

Suppose that $U_{E}^{F} \in C_{G}(\hat{D}, \hat{T})$. Let $m=1$ and $n=0$; we get that $G \cap \tilde{E} \subseteq \mathcal{D}_{E}^{F}$. 
Conversely, if $G \cap \tilde{E} \subseteq \mathcal{D}_{E}^{F}$, then, for any $n, m \in \mathbb{Z}, \forall s \in 2^{n} G \cap \tilde{E}$ (this implies that $\left.2^{-n} s \in G \cap \tilde{E}\right)$, there is an integer $k$ such that $2^{-n} s=\sigma_{E}^{F}\left(2^{-n} s\right)+2 k \pi$. So $\frac{m}{2^{n}} s=\frac{m}{2^{n}} \sigma_{E}^{F}(s)+2 k m \pi$. Thus $U_{E}^{F} \in C_{G}(\hat{D}, \hat{T})$ by the above argument.

To get some other conditions we need the following properties:

Lemma 2.3. Let $E$ and $F$ be two sub-frame sets. Then:

(i) $2 \mathcal{D}_{E}^{F} \subseteq \mathcal{D}_{E}^{F}$.

(ii) $\mathcal{D}_{E}^{F}=\bigcup_{k \in \mathbb{Z}} \bigcup_{n=[k]}^{\infty} 2^{n} E_{k}$, where $E_{k}=\left\{s \in E: \sigma_{E}^{F}(s)-s=2 k \pi\right\}$ and $[k]=\min \left\{-m \in \mathbb{Z}: m>0,2^{m} \mid k\right\}$.

(iii) $\mathcal{D}_{E}^{F} \supseteq \bigcup_{n=0}^{\infty} 2^{n} E$, and $\mathcal{D}_{E}^{F}=\bigcup_{n=0}^{\infty} 2^{n} E$ if and only if $\sigma_{E}^{F}(s)-s$ is an odd integral multiple of $2 \pi$ for $s \in E$ a.e.

(iv) $\mathcal{D}_{E}^{F}=\tilde{E}$ if and only if $E=F$.

(v) $\mathcal{D}_{E}^{F}=\sigma_{F}^{E}\left(\mathcal{D}_{F}^{E}\right)$.

(vi) $\mathcal{D}_{E}^{F} \sim_{2 \pi} \mathcal{D}_{F}^{E}$ and $\left(\mathcal{D}_{E}^{F} \backslash \bigcup_{n \geq 0} 2^{n} E\right) \sim_{2 \pi}\left(\mathcal{D}_{F}^{E} \backslash \bigcup_{n \geq 0} 2^{n} F\right)$.

(vii) $\mathcal{D}_{E}^{F}=\mathcal{D}_{F}^{E}$ if and only if $\sigma_{E}^{F}(s)-\sigma_{F}^{E}(s) \in 2 \mathbb{Z} \pi$ for all $s \in \mathcal{D}_{E}^{F} \cup \mathcal{D}_{F}^{E}$.

Proof. (i) follows from the definition of $\mathcal{D}_{E}^{F}$ and the fact that $\sigma_{E}^{F}(2 s)=2 \sigma_{E}^{F}(s)$.

(ii) Suppose that $t \in E_{k}$. Then $\sigma_{E}^{F}(t)-t=2 k \pi$. Since $2^{n} k \in \mathbb{Z}$ if $n \geq[k]$, we have that $2^{n} t \in \mathcal{D}_{E}^{F}$ because $\sigma_{E}^{F}\left(2^{n} t\right)-2^{n} t=2\left(2^{n} k\right) \pi$. Thus $\bigcup_{k \in \mathbb{Z}} \bigcup_{n=[k]}^{\infty} 2^{n} E_{k} \subseteq \mathcal{D}_{E}^{F}$.

Conversely, let $s \in \mathcal{D}_{E}^{F}$. Then $s \in 2^{n} E_{k}$ for some $n$ and $k$. This implies that $s=2^{n} t$ for some $t \in E_{k}$. Hence $\sigma_{E}^{F}\left(2^{n} t\right)-2^{n} t=2^{n}\left(\sigma_{E}^{F}(t)-t\right)=2^{n}(2 k \pi)$. Thus $2^{n} k \in \mathbb{Z}$ since $2^{n} t \in \mathcal{D}_{E}^{F}$, and so $s \in \bigcup_{k \in \mathbb{Z}} \bigcup_{n=[k]}^{\infty} 2^{n} E_{k}$.

(iii) By the definition of $\sigma_{E}^{F}$, we have $E \subset \mathcal{D}_{E}^{F}$. Thus the relation $\mathcal{D}_{E}^{F} \supseteq \bigcup_{n=0}^{\infty} 2^{n} E$ follows from (i).

The second part of (iii). By (ii), we have that $\mathcal{D}_{E}^{F}=\bigcup_{n=0}^{\infty} 2^{n} E$ holds if and only if $\mu\left(E_{k}\right)=0$ whenever $[k] \neq 0$, which, in turn, is equivalent to the fact that $\sigma_{E}^{F}(s)-s$ is an odd integral multiple of $2 \pi$ for $s \in E$, a.e.

(iv) By (ii), we get that $\mathcal{D}_{E}^{F}=\tilde{E}$ if and only if $E_{k}$ is a measure zero set whenever $k \neq 0$. That is, $E_{0}=E$. But $E_{0}=E$ is equivalent to $E=F$. Hence we get (iv).

(v) Let $s \in \mathcal{D}_{F}^{E}$. Then there is an integer $k$ satisfying $\sigma_{F}^{E}(s)-s=2 k \pi$. That is, $\sigma_{F}^{E}(s)-\sigma_{E}^{F}\left(\sigma_{F}^{E}(s)\right)=2 k \pi$. Thus $\sigma_{F}^{E}(s) \in \mathcal{D}_{E}^{F}$.

Similarly, for any $t \in \mathcal{D}_{E}^{F}$, we have $\sigma_{E}^{F}(t) \in \mathcal{D}_{F}^{E}$, which implies that $t=$ $\sigma_{F}^{E}\left(\sigma_{E}^{F}(t)\right) \in \sigma_{F}^{E}\left(\mathcal{D}_{F}^{E}\right)$. Thus we get $(\mathrm{v})$.

(vi) From (ii), we have

$$
\mathcal{D}_{E}^{F} \backslash \bigcup_{n \geq 0} 2^{n} E=\bigcup_{k \in \mathbb{Z}[k] \leq n<0} \bigcup^{n} E_{k}
$$

and

$$
\mathcal{D}_{F}^{E} \backslash \bigcup_{n \geq 0} 2^{n} F=\bigcup_{k \in \mathbb{Z}} \bigcup_{[k] \leq n<0} 2^{n} F_{k} .
$$

By the definition of $E_{k}$ and $F_{k}$, we also have

$$
2^{n} E_{k} \sim_{2 \pi} 2^{n} F_{-k}
$$

if $n \geq[k]$.

Thus $\mathcal{D}_{E}^{F} \sim_{2 \pi} \mathcal{D}_{F}^{E}$ and $\left(\mathcal{D}_{E}^{F} \backslash \bigcup_{n \geq 0} 2^{n} E\right) \sim_{2 \pi}\left(\mathcal{D}_{F}^{E} \backslash \bigcup_{n \geq 0} 2^{n} F\right)$.

(vii) Assume that $\mathcal{D}_{E}^{F}=\mathcal{D}_{F}^{E}$ and let $s \in \mathcal{D}_{E}^{F}$. Then $\sigma_{E}^{F}(s)-s \in 2 \mathbb{Z} \pi$. Since also $s \in \mathcal{D}_{F}^{E}$, we have that $\sigma_{F}^{E}(s)-s \in 2 \mathbb{Z} \pi$. Thus $\sigma_{E}^{F}(s)-\sigma_{F}^{E}(s) \in 2 \mathbb{Z} \pi$. 
Conversely, suppose that $\sigma_{E}^{F}(s)-\sigma_{F}^{E}(s) \in 2 \mathbb{Z} \pi$ for all $s \in \mathcal{D}_{E}^{F} \cup \mathcal{D}_{F}^{E}$ and let $s \in \mathcal{D}_{E}^{F}$. Then $\sigma_{E}^{F}(s)-\sigma_{F}^{E}(s) \in 2 \mathbb{Z} \pi$ and $\sigma_{E}^{F}(s)-s \in 2 \mathbb{Z} \pi$, which implies that $\sigma_{F}^{E}(s)-s \in 2 \mathbb{Z} \pi$, and so $s \in \mathcal{D}_{F}^{E}$. Similarly, $\mathcal{D}_{F}^{E} \subset \mathcal{D}_{E}^{F}$ and therefore $\mathcal{D}_{E}^{F}=\mathcal{D}_{F}^{E}$.

By Lemma 2.3 and Theorem 2.2, we immediately have the following:

Corollary 2.4. (i) $U_{E}^{F} \in C_{E}(\hat{D}, \hat{T})$.

(ii) $U_{F}^{E} \in C_{E}(\hat{D}, \hat{T}) \Leftrightarrow E \cap \tilde{F} \subset \mathcal{D}_{F}^{E}=\sigma_{E}^{F}\left(\mathcal{D}_{E}^{F}\right)$. In particular, if both $E$ and $F$ are wavelet sets, then $U_{F}^{E} \in C_{E}(\hat{D}, \hat{T}) \Leftrightarrow E \subset \mathcal{D}_{F}^{E}=\sigma_{E}^{F}\left(\mathcal{D}_{E}^{F}\right)$.

(iii) If $E \subset \bigcup_{n=0}^{\infty} 2^{n} F$, then $U_{E}^{F}, U_{F}^{E} \in C_{E}(\hat{D}, \hat{T})$.

In order to consider when $\left(U_{E}^{F}\right)^{k} \in C_{G}(\hat{D}, \hat{T})$, we need to extend Definition 2.1 to the following more general case. The $k$-th domain of $2 \pi$-congruence of $\sigma_{E}^{F}$ is the set of all points $s \in \tilde{E}$ for which $\left(\sigma_{E}^{F}\right)^{k}(s)-s$ is an integral multiple of $2 \pi$. We denote it by $\mathcal{D}_{E}^{F}(k)$.

Replacing $\sigma_{E}^{F}$ by $\left(\sigma_{E}^{F}\right)^{k}$ in the proof of Theorem 2.2, we get

Theorem 2.5. $\left(U_{E}^{F}\right)^{k} \in C_{G}(\hat{D}, \hat{T})$ if and only if $G \cap \tilde{E} \subseteq \mathcal{D}_{E}^{F}(k)$.

Corollary 2.6. The following are equivalent:

(i) $\left(U_{E}^{F}\right)^{2} \in C_{E}(\hat{D}, \hat{T})$.

(ii) $\sigma_{E}^{F}(s)-\sigma_{F}^{E}(s)$ is an integral multiple of $2 \pi, \forall s \in F$.

(iii) $F \subset \mathcal{D}_{E}^{F}$.

Proof. Suppose that $\left(U_{E}^{F}\right)^{2} \in C_{E}(\hat{D}, \hat{T})$. Then, by Theorem 2.5, $E=E \cap \tilde{E} \subset$ $\mathcal{D}_{E}^{F}(2)$. Thus for every $s \in E$, there is an integer $l$ such that $\left(\sigma_{E}^{F}\right)^{2}(s)-s=2 l \pi$, i.e. $\sigma_{E}^{F}\left(\sigma_{E}^{F}(s)\right)-\sigma_{F}^{E}\left(\sigma_{E}^{F}(s)\right)=2 l \pi$. Since $\sigma_{E}^{F}(E)=F$, we have that for each $s \in F, \sigma_{E}^{F}(s)-\sigma_{F}^{E}(s)$ is an integral multiple of $2 \pi$. Hence (i) implies (ii).

Now assume (ii). Then for each $s \in F$, we have both $\sigma_{E}^{F}(s)-\sigma_{F}^{E}(s) \in 2 \mathbb{Z} \pi$ and $\sigma_{F}^{E}(s)-s \in 2 \mathbb{Z} \pi$. Thus $\sigma_{E}^{F}(s)-s \in 2 \mathbb{Z} \pi$, which implies (iii).

For (iii) $\Rightarrow(\mathrm{i})$, take any $s \in E$. We have $\sigma_{E}^{F}(s) \in \mathcal{D}_{E}^{F}$ by the assumption and the fact that $\sigma_{E}^{F}(s) \in F$. So $\left(\sigma_{E}^{F}\right)^{2}(s)-\sigma_{E}^{F}(s) \in 2 \mathbb{Z} \pi$. Note that $s \in \mathcal{D}_{E}^{F}$. We get $\left(\sigma_{E}^{F}\right)^{2}(s)-s \in 2 \mathbb{Z} \pi$. Hence $s \in \mathcal{D}_{E}^{F}(2)$. By Theorem 2.5 we have $\left(U_{E}^{F}\right)^{2} \in$ $C_{E}(\hat{D}, \hat{T})$.

Let $E$ and $F$ be two wavelet sets. If $\left(U_{E}^{F}\right)^{2}=I$, then $(E, F)$ is called an interpolation pair. In this case the inverse Fourier transform $\frac{1}{\sqrt{2 \pi}}\left(a \chi_{E}+i b \chi_{F}\right)$ is always a wavelet when $a$ and $b$ are real numbers such that $a^{2}+b^{2}=1$. This can be generalized to function coefficient cases (see [DL]). If $(E, F)$ is an interpolation pair, then $\mathcal{D}_{E}^{F}=\mathcal{D}_{F}^{E}$ since $\sigma_{E}^{F}=\sigma_{F}^{E}$. Conversely, if $\mathcal{D}_{E}^{F}=\mathcal{D}_{F}^{E}$, then by (vii) of Lemma 2.3, (ii) of Corollary 2.4 and Corollary 2.6, we have $U_{E}^{F}, U_{F}^{E},\left(U_{E}^{F}\right)^{2} \in$ $C_{E}(\hat{D}, \hat{T})$. We ask:

Question. Does $\mathcal{D}_{E}^{F}=\mathcal{D}_{F}^{E}$ imply $\left(U_{E}^{F}\right)^{2}=I$ ?

Multiresolution analysis (MRA) developed by Mallat [Ma] and Meyer $\overline{\mathrm{Me}}$ is an important tool in constructing orthogonal wavelets and in applications such as signal and image processing. Recall that a multiresolution analysis (MRA) is a sequence $\left\{V_{j}: j \in \mathbb{Z}\right\}$ of closed subspaces of $L^{2}(\mathbb{R})$ satisfying:

(1) $V_{j} \subset V_{j+1}, j \in \mathbb{Z}$,

(2) $\bigcap_{j \in \mathbb{Z}} V_{j}=\{0\}, \overline{\bigcup_{j \in \mathbb{Z}} V_{j}}=L^{2}(\mathbb{R})$,

(3) $f \in V_{j}$ if and only if $D f \in V_{j+1}, j \in \mathbb{Z}$, 
(4) there is a scaling function $\phi \in V_{0}$ such that $\left\{T_{\ell} \phi: \ell \in \mathbb{Z}\right\}$ forms an orthonormal basis for $V_{0}$.

It is known (cf. $\mathrm{Dau}$ ) that there is an orthonormal wavelet $\psi$ in $V_{1} \ominus V_{0}$, which is called an MRA wavelet associated with $\left(V_{j}\right)$. Not every wavelet is an MRA wavelet. A wavelet set $E$ is called an $M R A$ wavelet set if the corresponding wavelet is an MRA wavelet. It is easy to check (cf. [HW]) that $E$ is an MRA wavelet set if and

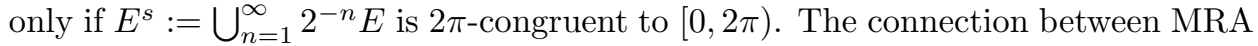
wavelet sets and the interpolation operators is given by the following result:

Theorem 2.7. Suppose that $E$ is an MRA wavelet set. Then $F$ is an MRA wavelet set if one of the following equivalent conditions is true:

(1) $U_{E}^{F} \in C_{F}(\hat{D}, \hat{T})$,

(2) $\left(U_{E}^{F}\right)^{2} \in C_{E}(\hat{D}, \hat{T})$.

Proof. By Corollary 2.4(ii) and Corollary 2.6, (1) and (2) are equivalent.

Assume that (1) (or equivalently (2)) holds. By the characterization of an MRA wavelet set, we first need to show that $\bigcup_{n \in \mathbb{Z}}\left(F^{s}+2 n \pi\right)=\mathbb{R}$. Since $E$ is an MRA wavelet set, $\left\{E^{s}+2 n \pi: n \in \mathbb{Z}\right\}$ forms a partition of $\mathbb{R}$. So it suffices to show that $2^{-j} x \in \bigcup_{n \in \mathbb{Z}}\left(F^{s}+2 n \pi\right)$ for every $x \in E$ and for all integer $j \geq 1$. Fix $x \in E$ and let $k$ be the unique integer such that $2^{k} x \in F$.

If $k \geq 0$, then clearly $2^{-j} x=2^{-(j+k)}\left(2^{k} x\right) \in F^{s}$ since $2^{k} x \in F$ and $j+k>0$.

Now assume that $k$ is negative and write $k=-l$ with $l>0$. Since $U_{E}^{F} \in$ $C_{F}(\hat{D}, \hat{T})$, then, by Corollary 2.4(ii), we have $F \subset \mathcal{D}_{E}^{F}$. Hence $\sigma_{E}^{F}\left(2^{-l} x\right)-2^{-l} x=$ $2 m \pi$ for some $m \in \mathbb{Z}$. Let $\sigma_{E}^{F}(x)=x+2 n \pi \in F$. Then

$$
2^{-l} x+2 m \pi=\sigma_{E}^{F}\left(2^{-l} x\right)=2^{-l} \sigma_{E}^{F}(x)=2^{-l}(x+2 n \pi) .
$$

This implies that $n=2^{l} m$.

There are two cases. Case (a): If $j>l$, then $2^{-j} x=2^{-(j-l)} x 2^{-l} x \in F^{s}$ since $j-l>0$ and $2^{-l} x \in F$. Case (b): If $1 \leq j \leq l$, then $2^{-j} n \in \mathbb{Z}$ since we know that $2^{-l} n=m \in \mathbb{Z}$. Thus $2^{-j} x=2^{-j}(x+2 n \pi)-2\left(2^{-j} n\right) \pi \in \bigcup_{i \in \mathbb{Z}}\left(F^{s}+2 i \pi\right)$ since we know that $x+2 n \pi \in F$ and $2^{-j} n \in \mathbb{Z}$.

Therefore we have covered all the cases and hence $2^{-j} x \in \bigcup_{i \in \mathbb{Z}}\left(F^{s}+2 i \pi\right)$ for all $x \in E$ and all integers $j>0$. Therefore $\bigcup_{n \in \mathbb{Z}}\left(F^{s}+2 n \pi\right) \supseteq \bigcup_{n \in \mathbb{Z}}\left(E^{s}+2 n \pi\right)=\mathbb{R}$. This implies that there exists a subset of $F^{S}$ which is $2 \pi$-congruent to $[0,2 \pi)$ (cf. Han]). Note that $F^{s}$ has measure $2 \pi$. Thus $F^{s}$ is $2 \pi$-congruent to $[0,2 \pi)$ which implies that $F$ is an MRA wavelet set.

In general we have the following:

Theorem 2.8. Let $E$ be an MRA wavelet set. Then a wavelet set $F$ is also an $M R A$ wavelet set if and only if $\left(\mathbb{R} \backslash \mathcal{D}_{E}^{F}\right) \sim_{2 \pi}\left(\mathbb{R} \backslash \mathcal{D}_{F}^{E}\right)$.

Proof. Since $E^{s}=\bigcup_{j<0} 2^{j} E, \mathbb{R}=\bigcup_{n \in \mathbb{Z}} E$ and $\mathcal{D}_{E}^{F}=\bigcup_{k \in \mathbb{Z}} \bigcup_{n \geq[k]} 2^{n} E_{k}$, it follows that $E^{s} \backslash \mathcal{D}_{E}^{F}=\mathbb{R} \backslash \mathcal{D}_{E}^{F}$. Similarly for $F, F^{s} \backslash \mathcal{D}_{F}^{E}=\mathbb{R} \backslash \mathcal{D}_{F}^{E}$. We also have

$$
\begin{aligned}
& E^{s}=\left(E^{s} \backslash \mathcal{D}_{E}^{F}\right) \cup\left(\mathcal{D}_{E}^{F} \backslash \bigcup_{n \geq 0} 2^{n} E\right), \\
& F^{s}=\left(F^{s} \backslash \mathcal{D}_{F}^{E}\right) \cup\left(\mathcal{D}_{F}^{E} \backslash \bigcup_{n \geq 0} 2^{n} F\right) .
\end{aligned}
$$


Now suppose that both $E$ and $F$ are MRA wavelet sets. Then $E^{s} \sim_{2 \pi}[0,2 \pi) \sim$ $F^{s}$. By (vi) of Lemma 2.3,

$$
\left(\mathcal{D}_{E}^{F} \backslash \bigcup_{n \geq 0} 2^{n} E\right) \sim_{2 \pi}\left(\mathcal{D}_{F}^{E} \backslash \bigcup_{n \geq 0} 2^{n} F\right) .
$$

Thus we have $\left(E^{s} \backslash \mathcal{D}_{E}^{F}\right) \sim_{2 \pi}\left(F^{S} \backslash \mathcal{D}_{F}^{E}\right)$.

Conversely, suppose that $\mathbb{R} \backslash \mathcal{D}_{E}^{F} \sim_{2 \pi} \mathbb{R} \backslash \mathcal{D}_{F}^{E}$. Then $E^{s} \sim_{2 \pi} F^{s}$ follows from $\left(\mathcal{D}_{E}^{F} \backslash \bigcup_{n \geq 0} 2^{n} E\right) \sim_{2 \pi}\left(\mathcal{D}_{F}^{E} \backslash \bigcup_{n \geq 0} 2^{n} F\right)$.

The following is an immediate corollary of Theorem 2.8 or Theorem 2.7.

Theorem 2.9. If $\mathcal{D}_{E}^{F}=\mathcal{D}_{F}^{E}$, then $E$ is an $M R A$ wavelet set if and only if $F$ is. In particular, this is true when $(E, F)$ is an interpolation pair.

The following example shows that $U_{E}^{F}$ may not be in $C_{F}(\hat{D}, \hat{T})$ for some subframe sets $E$ and $F$.

Example. Let $E=[2 \pi, 4 \pi)$ and let $F=\left[-3 \pi,-\frac{3 \pi}{2}\right) \cup\left[\frac{\pi}{2}, \pi\right)$ and

$$
G=\left[-\frac{\pi}{2},-\frac{\pi}{4}\right) \cup\left[\pi, \frac{5 \pi}{4}\right) \cup\left[\frac{7 \pi}{4}, 2 \pi\right) \cup\left[\frac{5 \pi}{2}, 3 \pi\right) \cup\left[\frac{13 \pi}{4}, \frac{7 \pi}{2}\right) \cup\left[6 \pi, \frac{13 \pi}{2}\right) .
$$

Then $F$ and $G$ are wavelet sets (see [DL]). A simple calculation shows that

$$
\sigma_{E}^{F}(s)= \begin{cases}s-4 \pi, & s \in\left[2 \pi, \frac{5 \pi}{2}\right), \\ s-2 \pi, & s \in\left[\frac{5 \pi}{2}, 3 \pi\right), \\ s-6 \pi, & s \in[3 \pi, 4 \pi),\end{cases}
$$

and

$$
\begin{aligned}
\mathcal{D}_{E}^{F} & =\left(\bigcup_{n=-1}^{\infty} 2^{n}\left[2 \pi, \frac{5 \pi}{2}\right)\right) \cup\left(\bigcup_{n=0}^{\infty} 2^{n}\left[\frac{5 \pi}{2}, 3 \pi\right)\right) \cup\left(\bigcup_{n=0} 2^{n}[3 \pi, 4 \pi)\right) \\
& =\left[\pi, \frac{5 \pi}{4}\right) \cup[2 \pi, \infty) .
\end{aligned}
$$

Similarly, we have

$$
\sigma_{F}^{E}(s)= \begin{cases}s+6 \pi, & s \in[-3 \pi,-2 \pi), \\ s+4 \pi, & s \in\left[-2 \pi,-\frac{3 \pi}{2}\right), \\ s+2 \pi, & s \in\left[\frac{\pi}{2}, \pi\right),\end{cases}
$$

and

$$
\mathcal{D}_{F}^{E}=\left(-\infty,-\frac{3 \pi}{2}\right) \cup\left[-\pi,-\frac{3 \pi}{4}\right) \cup\left[\frac{\pi}{2}, \infty\right) .
$$

Thus by Theorem 2.2 we have $U_{E}^{F}, U_{F}^{E} \notin C_{G}(\hat{D}, \hat{T})$ and $U_{E}^{F} \notin C_{F}(\hat{D}, \hat{T})$, but they both belong to $C_{E}(\hat{D}, \hat{T})$. So $U_{F}^{E}$ is a non-unitary isometry in $C_{F}(\hat{D}, \hat{T})$ with the property that $\left(U_{F}^{E}\right)^{*}$ is not in $C_{F}(\hat{D}, \hat{T})$. 
Now let $K=[-4 \pi,-2 \pi)$. Similarly we have

$$
\sigma_{K}^{F}(s)=\left\{\begin{aligned}
s+2 \pi, & s \in\left[-4 \pi,-\frac{7 \pi}{2}\right], \\
s+4 \pi, & s \in\left[-\frac{7 \pi}{2},-3 \pi\right), \\
s, & s \in[-3 \pi,-2 \pi),
\end{aligned}\right.
$$

and

$$
\begin{gathered}
D_{K}^{F}=(-\infty,-2 \pi) \cup\left[-\frac{7 \pi}{4},-\frac{3 \pi}{2}\right) \cup\left(\bigcup_{n<0} 2^{n}[-3 \pi,-2 \pi)\right), \\
\mathcal{D}_{F}^{K}=\left(-\infty,-\frac{3 \pi}{2}\right) \cup\left[\frac{\pi}{4}, \infty\right) \cup\left(\bigcup_{n<0} 2^{n}[-3 \pi,-2 \pi)\right) .
\end{gathered}
$$

Thus $E \cap \tilde{K} \phi \subset D_{K}^{F}$ and $E \cap \tilde{F}=E \subset \mathcal{D}_{F}^{K}$, which implies by Theorem 2.2 that $U_{K}^{F}$ and $U_{F}^{K}$ also belong to $C_{E}(\hat{D}, \hat{T})$.

Note that $U_{E}^{F} U_{F}^{E}+U_{K}^{F} U_{F}^{K}=I$, and $U_{F}^{E}, U_{F}^{K}$ have orthogonal ranges. Therefore $C_{E}(\hat{D}, \hat{T})$ contains the generators and their adjoints for the Cuntz algebra $\mathcal{O}_{2}$.

We conclude this paper by extending Theorem 5.2 in DL to the sub-frame set case. The proof is similar to that in $\mathrm{DL}$ and is left to the interested reader.

Theorem 2.10. Let $E$ and $F$ be sub-frame sets. Then:

(i) $U_{E}^{F}$ normalizes $\{\hat{D}, \hat{T}\}^{\prime}$, i.e. $U_{E}^{F}\{\hat{D}, \hat{T}\}^{\prime} U_{F}^{E} \subset\{\hat{D}, \hat{T}\}^{\prime}$;

(ii) if $E \neq F$, then $U_{E}^{F} \notin\{\hat{D}, \hat{T}\}^{\prime \prime}$.

Corollary 2.11. If $E$ is a sub-frame set, then $C_{E}(\hat{D}, \hat{T})$ is nonabelian.

Proof. Choose any sub-frame set $F$ such that $F \sim_{2 \pi} E$ and $E \neq F$. Then, by Corollary 2.4 and Theorem 2.10, $U_{E}^{F} \in C_{E}(\hat{D}, \hat{T})$ and $U_{E}^{F} \notin\{\hat{D}, \hat{T}\}^{\prime \prime}$. Note that $C_{E}(\hat{D}, \hat{T}) \supset\{\hat{D}, \hat{T}\}^{\prime}$. We obtain that $C_{E}(\hat{D}, \hat{T})$ is not abelian.

\section{REFERENCES}

[DL] X. Dai and D. Larson, Wandering vectors for unitary systems and orthogonal wavelets, Memoirs Amer. Math. Soc., 640 (1998). MR 98m:47067

[DLS] X. Dai, D. R. Larson and D. Speegle, Wavelets in $\mathbb{R}^{n}$, J. Fourier Analysis and Applications, 3 (1997), 451-456. MR 98m:42048

[DLu] X. Dai and S. Lu, Wavelet in subspaces, Michigan J. Math., 43 (1996), 81-98. MR 97m:42021

[DDG] X. Dai, Y. Diao and Q. Gu, Subspaces with normalized tight frames in $\mathbb{R}$, Proc. Amer. Math. Soc., 129 (2001), 2045-2055. MR 2002a:46006

[DDGH] X. Dai, Y. Diao, Q. Gu and D. Han, Frame wavelet sets in $\mathbb{R}^{d}$, Proc. Amer. Math. Soc., to appear.

[Dau] I. Daubechies, Ten Lectures on Wavelets, CBS-NSF Regional Conferences in Applied Mathematics, 61, SIAM (1992). MR 93e:42045

[Han] D. Han, Unitary systems, wavelets and operator algebras, Ph.D. thesis, Texas A\&M University, 1998.

[HL] D. Han and D. R. Larson, Frames, bases and group representations, Memoirs Amer. Math. Soc., 697 (2000). MR 2001a:47013

[HW] E. Hernandez and G. Weiss, A First Course in Wavelets, Studies in Advanced Mathematics, CRC Press, 1996. MR 97i:42015

[La] D. Larson, Von Neumann algebras and wavelets. Operator algebras and applications (Samos, 1996), NATO Adv. Sci. Inst. Ser. C Math. Phys. Sci., 495, Kluwer Acad. Publ., Dordrecht (1997), 267-312. MR 98g:46091 
[Ma] S. Mallat, Multiresolution approximations and wavelet orthonormal basis of $L^{2}(\mathbb{R})$, Trans. Amer. Math. Soc., 315 (1989), 69-87. MR 90e:42046

[Me] Y. Meyer, Ondelettes et Opeŕateurs, Herman, Paris, 1990. MR 93i:42002, MR 93i:42003

[Sp] D. Speegle, The s-elementary wavelets are connected, Proc. Amer. Math. Soc. 127(1999), 223-233. MR 99b:42045

Department of Mathematics, University of Central Florida, Orlando, Florida 32816

E-mail address: dhan@pegasus.cc.ucf.edu 